\title{
ON THE GROWTH OF THE SPHERICAL DERIVATIVE OF A MEROMORPHIC FUNCTION
}

\author{
SAKARI TOPPILA
}

\section{Introduction}

Let $f$ be meromorphic in the plane. We denote

and

$$
\begin{gathered}
\varrho(f(z))=\frac{\left|f^{\prime}(z)\right|}{1+|f(z)|^{2}}, \\
\mu(r, f)=\sup \{\varrho(f(z)):|z|=r\} \\
\lambda(r, f)=\inf \{\varrho(f(z)):|z|=r\} .
\end{gathered}
$$

In this paper, we shall give some estimates on the growth of $\mu(r, f)$ and $\lambda(r, f)$.

\section{On the growth of $\lambda(r, f)$}

We shall employ the usual notation of the Nevanlinna theory. First we shall estimate the growth of $\lambda(r, f)$ from below.

Theorem 1. Let $f$ be a transcendental meromorphic function of finite lower order. Then

$$
\limsup _{r \rightarrow \infty} \frac{\log \lambda(r, f)}{T(r, f)}>-\infty .
$$

This result need not hold for functions of infinite lower order. If we take $f(z)=$ $\exp \left\{e^{z}\right\}$, then

$$
\log \lambda(r, f) \leqq \log \varrho(f(r)) \leqq-(1+o(1)) e^{r}
$$

and $T(r, f)=o\left(e^{r}\right)$ as $r \rightarrow \infty$. These estimates imply that

$$
\limsup _{r \rightarrow \infty} \frac{\log \lambda(r, f)}{T(r, f)}=-\infty
$$


If $f$ is a meromorphic function of lower order zero, then $f$ satisfies

$$
\liminf _{r \rightarrow \infty} \frac{T(2 r, f)}{T(r, f)}=1
$$

For this class of functions we prove

Theorem 2. Let $f$ be a transcendental meromorphic function satisfying (2.3). Then

$$
\limsup _{r \rightarrow \infty} \frac{\log \lambda(r, f)}{T(r, f)} \geqq-1
$$

This does not hold for all functions of positive order. If we take $f(z)=e^{z}$, then

and since

$$
\lambda(r, f) \leqq \varrho(f(r)) \leqq e^{-r},
$$

$$
T(r, f)=(1+o(1)) \frac{r}{\pi}
$$

we deduce that

$$
\limsup _{r \rightarrow \infty} \frac{\log \lambda(r, f)}{T(r, f)} \leqq-\pi<-1
$$

for this function $f$.

In the other direction we have

Theorem 3. Let $f$ be a transcendental meromorphic function of finite order. Then

$$
\limsup _{r \rightarrow \infty} \frac{\log \lambda(r, f)}{T(r, f)} \leqq-\delta(\infty, f) .
$$

Theorems 2 and 3 together show that if $f$ is an entire transcendental function of finite order satisfying (2.3), then

$$
\limsup _{r \rightarrow \infty} \frac{\log \lambda(r, f)}{T(r, f)}=-1 .
$$

The condition (2.6) does not hold for all meromorphic functions of infinite order.

Theorem 4. There exists an entire function of infinite order and of lower order zero such that

$$
\limsup _{r \rightarrow \infty} \frac{\log \lambda(r, f)}{T(r, f)}=\infty
$$

For meromorphic functions without Nevanlinna deficient values the following theorem gives in some cases a sharper estimate than (2.6). 
Theorem 5. Let $f$ be a transcendental meromorphic function. Then

$$
\limsup _{r \rightarrow \infty} \frac{r \lambda(r, f)}{T(2 r, f)}<\infty
$$

On the other hand, we have

Theorem 6. Given any increasing and positive function $\varphi(r)$ such that $\varphi(r) \rightarrow \infty$ as $r \rightarrow \infty$, there exists a transcendental meromorphic function $f$ satisfying

$$
T(r, f)=O\left(\varphi(r)(\log r)^{2}\right) \text { as } r \rightarrow \infty
$$

such that

$$
\limsup _{r \rightarrow \infty} \frac{r \lambda(r, f)}{T(2 r, f)}>0
$$

and that

$$
\limsup _{r \rightarrow \infty} \frac{r \lambda(r, f)}{T(r, f)}=\infty
$$

The function $\varphi(r)$ in (2.10) cannot be replaced by a positive constant. We have

Theorem 7. If $f$ is a transcendental meromorphic function satisfying

$$
T(r, f)=O\left((\log r)^{2}\right) \text { as } r \rightarrow \infty,
$$

then

$$
\limsup _{r \rightarrow \infty} \frac{r \lambda(r, f)}{T(r, f)}=0
$$

\section{On the growth of $\mu(r, f)$}

The following theorem gives a lower bound for the growth of $\mu(r, f)$.

Theorem 8. Let $f$ be a transcendental meromorphic function. Then

$$
\liminf _{r \rightarrow \infty} \frac{\log \mu(r, f)}{T(r, f)} \geqq-1
$$

In the other direction, we have

Theorem 9. Let $f$ be a transcendental meromorphic function satisfying (2.3). Then

$$
\liminf _{r \rightarrow \infty} \frac{\log \mu(r, f)}{T(r, f)} \leqq-\delta(\infty, f)
$$


Combining Theorems 8 and 9, we deduce that

$$
\liminf _{r \rightarrow \infty} \frac{\log \mu(r, f)}{T(r, f)}=-1
$$

for transcendental entire functions satisfying (2.3).

The following theorem shows that (3.2) need not hold if the order of $f$ is positive.

Theorem 10. Given $d, 0<d<1$, there exists a meromorphic function $f$ of order $d$ with $\delta(\infty, f)>0$ such that

$$
\liminf _{r \rightarrow \infty} \frac{r \mu(r, f)}{T(r, f)}>0 .
$$

On the other hand, the following theorem shows that a transcendental meromorphic function of lower order zero cannot satisfy (3.4).

Theorem 11. Let $f$ be a transcendental meromorphic function. Then

$$
\liminf _{r \rightarrow \infty} \frac{r \mu(r, f)}{T(2 r, f)}<\infty,
$$

and if the lower order of $f$ is finite, then

$$
\liminf _{r \rightarrow \infty} \frac{r \mu(r, f)}{T(r, f)}<\infty .
$$

If, further, the lower order of $f$ is zero, then

$$
\liminf _{r \rightarrow \infty} \frac{r \mu(r, f)}{T(r, f)}=0 .
$$

If the order of $f$ is infinite, then (3.6) need not hold. We take

$$
f(z)=\exp \left\{i e^{z}\right\}
$$

Then $f^{\prime}(z)=i e^{z} f(z)$, and since $|f(z)|=1$ on the positive real axis, we get

$$
\mu(r, f) \geqq \frac{1}{2} e^{r} .
$$

Since

we get from (3.8)

$$
T(r, f)=o\left(e^{r}\right) \text { as } \quad r \rightarrow \infty,
$$

$$
\liminf _{r \rightarrow \infty} \frac{\mu(r, f)}{T(r+K, f)}=\infty
$$

for any positive constant $K$. 


\section{Proof of Theorem 1}

We use Lemma 3 of Hayman [4] in the following form.

Lemma A. Let $a_{n}, n=1, \ldots, p$, lie in $0<|z|<\infty$. For any $B \geqq 9$, there exists a set $E$ which is a countable union of discs $\left|z-c_{k}\right|<d_{k}$ such that

$$
\sum \frac{d_{k}}{\left|c_{k}\right|}<4000 e^{-B}
$$

and that

$$
\sum_{|z| / 2<\left|a_{n}\right|<2|z|} \log \frac{|z|+\left|a_{n}\right|}{\left|z-a_{n}\right|} \leqq p B
$$

when $z \neq 0$ and $z$ lies outside $E$.

Let $f$ be meromorphic in the plane and let $a_{n}$ be the $a$-points of $f$. We write

$$
S(z, a)=\sum_{|z| / 2<\left|a_{n}\right|<2|z|} \log \frac{|z|+\left|a_{n}\right|}{\left|z-a_{n}\right|} .
$$

Lemma 1. Let $f$ be a non-constant meromorphic function. Then for any complex value $a$ and any $B \geqq 12$ there exists a set $E$ which is a countable union of discs $\left|z-c_{k}\right|<$ $d_{k}$ such that

$$
\sum_{r<\left|c_{k}\right|<2 r} \frac{d_{k}}{\left|c_{k}\right|}<16000 e^{-B}
$$

for all positive $r$ and that

$$
S(z, a) \leqq n(4|z|, a, f) B
$$

when $z \neq 0$ and $z$ lies outside $E$.

Proof. It follows from Lemma A that

$$
S(z, a) \leqq n\left(2^{k+1}, a, f\right) B \leqq n(4|z|, a, f) B
$$

if $2^{k-1}<|z| \leqq 2^{k}$ and $z$ lies outside a set $E_{k}$ satisfying (4.1). We select from each $E_{k}$ those discs which have at least one common point with the annulus $2^{k-1}<|z|<2^{k}$ and denote the union of these discs by $E$. It follows from (4.5) that $S(z, a)$ satisfies (4.4) outside $E$ when $z \neq 0$. Since $B \geqq 12$, it follows from (4.1) that all discs which are selected from $E_{k}$ are contained in $2^{k-2}<|z|<2^{k+1}$, and (4.3) follows from (4.1). Lemma 1 is proved.

Let $f$ be as in Lemma 1. We choose $B=20$ and $a=\infty$ in Lemma 1, and denote the corresponding exceptional set $E$ by $E$.

Lemma 2. With the above notation we have

$$
\log |f(z)| \leqq 37 T(8|z|, f)
$$

when $|z| \geqq 1$ and $z$ lies outside $E$, where $E$ satisfies (4.3) with $B=20$. 
Proof. Let $b_{k}$ be the poles of $f$ and $|z| \geqq 1$. Applying the Poisson-Jensen formula with $R=2|z|$, we get

If $R / 4<\left|b_{k}\right|<R$, then

$$
\log |f(z)| \leqq 3 m(R, f)+\sum_{\left|b_{k}\right|<R} \log \left|\frac{R^{2}-\bar{b}_{k} z}{R\left(z-b_{k}\right)}\right| .
$$

$$
\log \left|\frac{R^{2}-\bar{b}_{k} z}{R\left(z-b_{k}\right)}\right| \leqq \log \frac{|z|+\left|b_{k}\right|}{\left|z-b_{k}\right|}+\log 8,
$$

and for other terms in the sum of (4.7) we get the upper bound $\log 8<3$. These estimates together with (4.7) imply that

$$
\log |f(z)| \leqq 3 T(2|z|, f)+3 n(2|z|, \infty, f)+S(z, \infty),
$$

and we deduce from Lemma 1 that

$$
\log |f(z)| \leqq 3 T(2|z|, f)+23 n(4|z|, \infty, f)
$$

when $|z| \geqq 1$ and $z$ lies outside $E$. Since

$$
n(r, \infty, f) \log 2 \leqq \int_{r}^{2 r} n(t, \infty, f) t^{-1} d t=N(2 r, \infty, f)-N(r, \infty, f) \leqq T(2 r, f)
$$

for $r \geqq 1$, (4.6) follows from (4.8). Lemma 2 is proved.

Now we prove Theorem 1. Let $f$ be a transcendental meromorphic function of finite lower order. Then there exist $K_{1}>0$ and a sequence $r_{n}, r_{n} \rightarrow \infty$ as $n \rightarrow \infty$, such that

for all $n$. Since

$$
T\left(32 r_{n}, f\right)<K_{1} T\left(r_{n}, f\right)
$$

$$
m\left(r, f^{\prime}\right) \leqq m(r, f)+m\left(r, f^{\prime} \mid f\right),
$$

we deduce from the lemma on the logarithmic derivative [Nevanlinna 7 p. 245] that

$$
m\left(16 r_{n}, f^{\prime}\right) \leqq m\left(16 r_{n}, f\right)+o\left(T\left(32 r_{n}, f\right)\right) \text { as } n \rightarrow \infty,
$$

which together with the fact that $N\left(r, f^{\prime}\right) \leqq 2 N(r, f)$ implies that

$$
T\left(16 r_{n}, f^{\prime}\right) \leqq(2+o(1)) T\left(32 r_{n}, f\right) \text { as } n \rightarrow \infty .
$$

Using Lemma 2, we deduce that there exists $t_{n}, r_{n} \leqq t_{n}<2 r_{n}$, such that

and

$$
\log |f(z)| \leqq O(T(8|z|, f))=O\left(T\left(16 r_{n}, f\right)\right)
$$

$$
-\log \left|f^{\prime}(z)\right| \leqq O\left(T\left(8|z|, 1 / f^{\prime}\right)\right)=O\left(T\left(16 r_{n}, f^{\prime}\right)\right)
$$

for all $z$ lying on $|z|=t_{n}$, and we deduce from (4.9) and (4.10) that

$$
-\log \lambda\left(t_{n}, f\right) \leqq O\left(T\left(32 r_{n}, f\right)\right)=O\left(T\left(t_{n}, f\right)\right) \quad \text { as } n \rightarrow \infty,
$$

which proves Theorem 1 . 


\section{Some properties of functions satisfying (2.3)}

We denote by $f^{(k)}$ the $k$-th derivative of $f$.

Lemma 3. Let $f$ be a transcendental meromorphic function satisfying (2.3), and let $k$ be a positive integer. Then there exists a sequence $r_{p}, r_{p} \rightarrow \infty$ as $p \rightarrow \infty$, such that

$$
T\left(p^{2} r_{p}, f^{(k)}\right)=(1+o(1)) T\left(r_{p}, f\right) \text { as } p \rightarrow \infty
$$

and

$$
T\left(p^{2} r_{p}, f^{(k)}\right)=T\left(r_{p}, f^{(k)}\right)+o\left(T\left(r_{p}, f\right)\right)
$$

as $p \rightarrow \infty$, and that for any complex value $a$

and

$$
\begin{gathered}
n\left(p^{2} r_{p}, a, f\right)=o\left(T\left(r_{p}, f\right)\right), \\
N\left(p^{2} r_{p}, a, f\right)=N\left(r_{p}, a, f\right)+o\left(T\left(r_{p}, f\right)\right), \\
n\left(p^{2} r_{p}, a, f^{(k)}\right)=o\left(T\left(r_{p}, f\right)\right)
\end{gathered}
$$

as $p \rightarrow \infty$.

Proof. It follows from (2.3) that there exists a sequence $t_{n}, t_{n} \rightarrow \infty$ as $n \rightarrow \infty$, such that

$$
T\left(2 t_{n}, f\right)=(1+o(1)) T\left(t_{n}, f\right) \text { as } n \rightarrow \infty .
$$

We choose $r_{1}=2$. Let $p \geqq 2$ be a positive integer and

$$
x=\frac{1}{p^{4} e^{2 p}} .
$$

Since $T(r, f)$ is an increasing and convex function of $\log r$, we get

$$
T\left(t_{n}, f\right)-T\left(x t_{n}, f\right) \leqq \frac{\log (1 / x)}{\log 2}\left(T\left(2 t_{n}, f\right)-T\left(t_{n}, f\right)\right),
$$

and we deduce from (5.7) that

$$
T\left(t_{n}, f\right)=(1+o(1)) T\left(x t_{n}, f\right) \text { as } n \rightarrow \infty .
$$

We choose $n$ so large that $x t_{n}>p^{2} r_{p-1}$ and

$$
T\left(t_{n}, f\right)<2 T\left(x t_{n}, f\right)
$$

and we set $r_{p}=x t_{n}$.

Let $a$ be a complex value. We get from (5.8)

$$
n\left(e^{p} r_{p}, a, f\right) p \leqq \int_{e^{p} r_{p}}^{e^{2 p} r_{p}} n(t, a, f) t^{-1} d t \leqq(1+o(1)) T\left(e^{2 p_{i}^{1} r_{p}}, f\right) \leqq(2+o(1)) T\left(r_{p}, f\right),
$$


which proves (5.3). From (5.9) we get

$$
N\left(p^{2} r_{p}, a, f\right)-N\left(r_{p}, a, f\right) \leqq 2 n\left(p^{2} r_{p}, a, f\right) \log p \leqq \frac{1}{p}(4+o(1)) T\left(r_{p}, f\right) \log p,
$$

which proves (5.4). If we choose $a$ such that $\Delta(a, f)=0$, we get from (5.4)

$$
\begin{aligned}
T\left(p^{2} r_{p}, f\right)-T\left(r_{p}, f\right)= & N\left(p^{2} r_{p}, a, f\right)-N\left(r_{p}, a, f\right)+o\left(T\left(p^{2} r_{p}, f\right)\right) \\
& =o\left(T\left(p^{2} r_{p}, f\right)\right),
\end{aligned}
$$

which proves (5.1).

It follows from the lemma on the logarithmic derivative that

$$
\begin{gathered}
T\left(t_{n} / 2, f^{(k)}\right) \leqq(k+1) N\left(t_{n} / 2, \infty, f\right)+m\left(t_{n} / 2, \infty, f\right)+m\left(t_{n} / 2, \infty, f^{(k)} / f\right) \\
\leqq(k+1+o(1)) T\left(t_{n}, f\right),
\end{gathered}
$$

and we deduce from (5.8) that

$$
T\left(t_{n} / 2, f^{(k)}\right) \leqq(2 k+2+o(1)) T\left(r_{p}, f\right)
$$

as $p \rightarrow \infty$. Just as in the proof of (5.9), we deduce from (5.12) that

$$
n\left(e^{p} r_{p}, a, f^{(k)}\right) \leqq \frac{1}{p}(1+o(1)) T\left(e^{2 p} r_{p}, f^{(k)}\right) \leqq \frac{1}{p}(2 k+2+o(1)) T\left(r_{p}, f\right),
$$

which proves (5.5), and just as in the proof of (5.10) and (5.11), we deduce that (5.6) and (5.2) follow from (5.13). Lemma 3 is proved.

We choose $B=20$ in Lemma 1 , and deduce that there exist sets $E_{1}, E_{2}, E_{3}$ and $E_{4}$ each of them satisfying (4.3) such that

$$
S(z, \infty, f)=S(z, \infty) \leqq 20 n(4|z|, \infty, f)
$$

outside $E_{1}$, and that corresponding estimates hold for $S(z, 0, f), S\left(z, \infty, f^{(k)}\right)$ and $S\left(z, 0, f^{(k)}\right)$ outside the union of $E_{2}, E_{3}$ and $E_{4}$. We write

$$
E=\bigcup_{k=1}^{4} E_{k} \text {. }
$$

Let $z=r e^{i \varphi}$ lie in $r_{p} \leqq|z| \leqq p r_{p}$. Let $b_{s}$ be the poles of $f$. Applying the PoissonJensen formula with $R=p^{2} r_{p}$, we get

$$
\begin{aligned}
\log |f(z)| & \leqq \frac{p+1}{p-1} m(R, \infty, f)-\frac{p-1}{p+1} m(R, 0, f) \\
& +\sum_{\left|b_{s}\right|<R} \log \left|\frac{R^{2}-\bar{b}_{s} z}{R\left(z-b_{s}\right)}\right| .
\end{aligned}
$$


If $|z| / 2<\left|b_{s}\right|<2|z|$, then

$$
\log \left|\frac{R^{2}-\bar{b}_{s} z}{R\left(z-b_{s}\right)}\right| \leqq \log \frac{|z|+\left|b_{s}\right|}{\left|z-b_{s}\right|}+\log \left(2 p^{2}\right),
$$

and for other terms in the sum of (5.15) we get the upper bound $\log \left(4 p^{2}\right)$. These estimates together with (5.15) imply that

$$
\begin{aligned}
\log |f(z)| & \leqq \frac{p+1}{p-1} m(R, \infty, f)-\frac{p-1}{p+1} m(R, 0, f) \\
& +S(z, \infty)+n(R, \infty, f) \log \left(4 p^{2}\right),
\end{aligned}
$$

and we deduce from (5.14) that

$$
\begin{aligned}
\log |f(z)| & \leqq N(R, 0, f)-N(R, \infty, f) \\
& +2\left(\frac{p+1}{p-1}-1+o(1)\right) T(R, f) \\
& +n(R, \infty, f)\left(20+\log \left(4 p^{2}\right)\right)
\end{aligned}
$$

if $z$ lies outside $E$. Therefore we get from (5.1), (5.4) and (5.9)

$$
\log |f(z)| \leqq N\left(r_{p}, 0, f\right)-N\left(r_{p}, \infty, f\right)+o\left(T\left(r_{p}, f\right)\right)
$$

for all $z$ lying in $r_{p} \leqq|z| \leqq p r_{p}$ outside $E$. A similar estimate holds for $1 / f$, and using (5.13) and Lemma 3 we get a similar estimate for $1 / f^{(k)}$ and $f^{(k)}$. We have proved the following result.

Lemma 4. Let $f$ and $k$ be as in Lemma 3. The sequence $r_{p}$ in Lemma 3 can be chosen such that

$$
\log |f(z)|=N\left(r_{p}, 0, f\right)-N\left(r_{p}, \infty, f\right)+o\left(T\left(r_{p}, f\right)\right)
$$

and

$$
\log \left|f^{(k)}(z)\right|=N\left(r_{p}, 0, f^{(k)}\right)-N\left(r_{p}, \infty, f^{(k)}\right)+o\left(T\left(r_{p}, f\right)\right)
$$

as $z \rightarrow \infty$ through the union of the annuli $r_{p} \leqq \mid z_{\mid} \leqq p r_{p}$ outside a set $E$ which is the union of four sets satisfying (4.3) with $B=20$.

\section{Proof of Theorem 2}

Let $f$ be as in Theorem 2. We may suppose that $f(0)=0$, because in other cases we can consider $1 / f$ or the function

Let

$$
\frac{f(z)-f(0)}{1+\overline{f(0)} f(z)}
$$

$$
f(z)=c_{k} z^{k}+c_{k+1} z^{k+1}+\ldots
$$


be the Laurent expansion of $f$ at the origin. Then $k \geqq 1$, and from (6.1) we deduce that there exists $t, 0<t<1$, such that if $0<|a|<t$, then

and

$$
n\left(\left(|a| /\left(2\left|c_{k}\right|\right)\right)^{1 / k}, a, f\right)=0
$$

$$
n\left(\left(2|a| /\left|c_{k}\right|\right)^{1 / k}, a, f\right)=k .
$$

These estimates imply that if $0<|a|<t$ and

then

$$
r>9\left(1+1 /\left|c_{k}\right|\right)=s_{0},
$$

$$
|\log | a||-N(r, a) \leqq|\log | a||-k \log \left(r\left(\left|c_{k}\right| /(2|a|)\right)^{1 / k}\right) \leqq \log \frac{2|a|}{r^{k}\left|c_{k}\right||a|} \leqq 0
$$

Let $0<|a|<9$. It follows from the first main theorem of the Nevanlinna theory that

$$
m(r, a) \leqq T(r, f)-N(r, a)+|\log | a||+\log +|a|+\log 2,
$$

and we deduce from (6.2) that

$$
m(r, a) \leqq T(r, f)+\log 2+\log (1 / t)+2 \log 9 \leqq T(r, f)+6+\log (1 / t)
$$

if $0<|a|<9$ and $r>s_{0}$.

We write

$$
M(r, f)=\sup \{|f(z)|:|z|=r\} .
$$

We apply Lemma 4 with $f^{(k)}=f^{\prime}$, and choose a sequence $r_{p}$ as in Lemmas 3 and 4 . We may assume that the circle $|z|=r_{p}$ lies outside the exceptional set $E$ of Lemma 4, because in other cases we may choose $r_{p}^{\prime}, r_{p}<r_{p}^{\prime}<2 r_{p}$, such that the circle $|z|=r_{p}^{\prime}$ lies outside $E$, and consider $\lambda\left(r_{p}^{\prime}, f\right)$ instead of $\lambda\left(r_{p}, f\right)$.

Let $z_{p}, f\left(z_{p}\right) \neq 0$, lie on $|z|=r_{p}$. We get for any $z$ lying on $|z|=r_{p}$

It follows from (5.18) that

$$
\left|f(z)-f\left(z_{p}\right)\right|=\left|\int_{z_{p}}^{z} f^{\prime}(w) d w\right| \leqq \pi r_{p} M\left(r_{p}, f^{\prime \prime}\right) .
$$

$$
\log \left|f^{\prime}(z)\right|=\log M\left(r_{p}, f^{\prime}\right)+o\left(T\left(r_{p}, f\right)\right)
$$

on $|z|=r_{F}$, and from (5.17) we get

$$
\log |f(z)|=\log \left|f\left(z_{p}\right)\right|+o\left(T\left(r_{p}, f\right)\right)
$$

for all $z$ lying on $|z|=r_{p}$.

We consider first those values of $p$ for which $\left|f\left(z_{p}\right)\right|<4$. From (6.4) we deduce that

$$
m\left(r_{p}, f\left(z_{p}\right), f\right) \geqq-\log M\left(r_{p}, f^{\prime}\right)-\log \left(\pi r_{p}\right),
$$


which implies together with (6.3) that

$$
\log M\left(r_{p}, f^{\prime}\right) \geqq-T\left(r_{p}, f\right)+o\left(T\left(r_{p}, f\right)\right) .
$$

Combining this with (6.5) and (6.6) we get

$$
\log \lambda\left(r_{p}, f\right) \geqq \log M\left(r_{p}, f^{\prime}\right)+o\left(T\left(r_{p}, f\right)\right) \geqq-T\left(r_{p}, f\right)+o\left(T\left(r_{p}, f\right)\right),
$$

which proves Theorem 2 in the case where $\left|f\left(z_{p}\right)\right|<4$ for an infinite number of values of $p$.

Let us suppose that $\left|f\left(z_{p}\right)\right| \geqq 4$. Applying the Jensen formula we get

$$
\log \left|f(0)-f\left(z_{p}\right)\right| \leqq \frac{1}{2 \pi} \int_{0}^{2 \pi} \log \left|f\left(z_{p}\right)-f\left(r_{p} e^{i \alpha}\right)\right| d \alpha+N\left(r_{p}, \infty, f\right),
$$

and since $f(0)=0$, it follows from (6.4) that

$$
\log \left|f\left(z_{p}\right)\right| \leqq \log M\left(r_{p}, f^{\prime}\right)+N\left(r_{p}, \infty, f\right)+o\left(T\left(r_{p}, f\right)\right) .
$$

This implies together with (6.5) and (6.6) that

$$
\begin{aligned}
\log \lambda\left(r_{p}, f\right) & \geqq \log M\left(r_{p}, f^{\prime}\right)-2 \log M\left(r_{p}, f\right)+o\left(T\left(r_{p}, f\right)\right) \\
& \geqq-N\left(r_{p}, \infty, f\right)-\log \left|f\left(z_{p}\right)\right|+o\left(T\left(r_{p}, f\right)\right),
\end{aligned}
$$

and using (5.17) we deduce that

$$
\log \lambda\left(r_{p}, f\right) \geqq-T\left(r_{p}, f\right)+o\left(T\left(r_{p}, f\right)\right) .
$$

This completes the proof of Theorem 2 .

\section{Proof of Theorem 3}

Let $f$ be as in Theorem 3. Let $z$ lie on the circle $|z|=r$. Since

we get

$$
\log ^{+}|f(z)| \leqq \log \left(\left|\frac{f^{\prime}(z)}{f(z)}\right| \frac{1+|f(z)|^{2}}{\left|f^{\prime}(z)\right|}\right) \leqq \log ^{+}\left|\frac{f^{\prime}(z)}{f(z)}\right|-\log \lambda(r, f),
$$

$$
m(r, f) \leqq m\left(r, f^{\prime} \mid f\right)-\log \lambda(r, f) .
$$

Since $f$ has finite order, it follows from the lemma on the logarithmic derivative that

$$
m\left(r, f^{\prime} \mid f\right)=o(T(r, f)) \text { as } r \rightarrow \infty,
$$

and we deduce from (7.1) that

$$
\log \lambda(r, f) \leqq-m(r, f)+o(T(r, f)) \leqq(-\delta(\infty, f)+o(1)) T(r, f) \quad \text { as } \quad r \rightarrow \infty .
$$

This proves Theorem 3 . 


\section{Proof of Theorem 4}

We set

$$
f(z)=\sum_{n=1}^{\infty}\left(z / r_{n}\right)^{s_{n}}
$$

where $r_{n}=\log s_{n}, s_{1}=100$, and for $n>1, s_{n}$ is a positive integer such that

$$
\log \log s_{n}>s_{n-1} \text {. }
$$

Then

It follows from (8.1) that

$$
f^{\prime}(z)=\sum_{n=1}^{\infty}\left(s_{n} / r_{n}\right)\left(z / r_{n}\right)^{s_{n}-1}
$$

$$
\log M\left(r_{n}, f\right) \leqq s_{n-1} \log r_{n} \leqq\left(\log r_{n}\right)^{2},
$$

which implies that the lower order of $f$ is zero. For $|z|=r_{n}$ it follows from (8.1) that

$$
\log \left|f^{\prime}(z)\right| \geqq \log \left(\frac{s_{n}}{r_{n}}-\sum_{k=1}^{n-1} s_{k} r_{n}^{s_{k}}\right) \geqq(1+o(1)) \log s_{n}=(1+o(1)) r_{n},
$$

and we deduce from (8.2) that

$$
\frac{\log \lambda\left(r_{n}, f\right)}{\log M\left(r_{n}, f\right)} \geqq \frac{(1+o(1)) r_{n}}{\left(\log r_{n}\right)^{2}} \rightarrow \infty \quad \text { as } \quad n \rightarrow \infty .
$$

This proves Theorem 4.

\section{Proof of Theorem 5}

Let $f$ be a transcendental meromorphic function. We write

$$
n(r)=n(r, 0, f)+n(r, 1, f)+n(r, \infty, f) .
$$

We have

$$
n(3 r / 2) \log (4 / 3) \leqq \int_{3 r / 2}^{2 r} n(t) t^{-1} d t \leqq(3+o(1)) T(2 r, f) \leqq 4 T(2 r, f)
$$

for $r \geqq r_{0}$. This implies that for all large values of $r$ we may choose $z_{r}$ lying on $|z|=r$ such that $f$ does not take any of the values 0,1 and $\infty$ in the disc

$$
\left|z-z_{r}\right|<\frac{r}{16 T(2 r, f)}
$$

It follows from Schottky's theorem that there exists an absolute constant $K_{1}$ such that if $\left|f\left(z_{r}\right)\right| \leqq 1$, then

$$
|f(z)|<K_{1}
$$


in

$$
D_{r}=\left\{z:\left|z-z_{r}\right|<\frac{r}{32 T(2, f)}\right\},
$$

and if $\left|f\left(z_{r}\right)\right|>1$, then $1 / f$ satisfies (9.3) in $D_{r}$.

We write $g(z)=f(z)$ if $\left|f\left(z_{r}\right)\right| \leqq 1$ and $g(z)=1 / f(z)$ if $\left|f\left(z_{r}\right)\right|>1$. Integrating along the boundary of $D_{r}$ we get from (9.3)

which implies that

$$
\left|g^{\prime}\left(z_{r}\right)\right|=\left|\frac{1}{2 \pi} \int \frac{g(w)}{\left(z_{r}-w\right)^{2}} d w\right| \leqq \frac{32 T(2 r, f) K_{1}}{r},
$$

$$
\frac{\left|f^{\prime}\left(z_{r}\right)\right|}{1+\left|f\left(z_{r}\right)\right|^{2}}=\frac{\left|g^{\prime}\left(z_{r}\right)\right|}{1+\left|g\left(z_{r}\right)\right|^{2}} \leqq \frac{32 K_{1} T(2 r, f)}{r},
$$

and we deduce that

$$
\frac{r \lambda(r, f)}{T(2 r, f)} \leqq 32 K_{1}
$$

for all large values of $r$. This proves Theorem 5 .

\section{Proof of Theorem 6}

It does not mean any restriction to assume that the function $\varphi(r)$ given in Theorem 6 satisfies the condition

$$
\varphi(r)=o(\log \log r) \quad \text { as } \quad r \rightarrow \infty .
$$

We choose $s_{1}=8, r_{1}=100$, and for $n \geqq 2, s_{n}$ and $r_{n}$ are chosen such that $s_{n}$ is a positive integer,

$$
\log \log r_{n}>r_{n-1},
$$

$$
s_{n}>8 n s_{n-1} \log r_{n}
$$

and

$$
s_{n}<\varphi\left(\sqrt{r_{n}}\right) \log r_{n} \text {. }
$$

We set

and

$$
f_{n}(z)=\frac{(-1)^{n}\left(z / r_{n}\right)^{s_{n}}}{1+\left(z / r_{n}\right)^{s_{n}}}
$$

$$
f(z)=\sum_{n=1}^{\infty} f_{n}(z) .
$$

Then

$$
f_{n}^{\prime}(z)=\frac{(-1)^{n}\left(s_{n} / r_{n}\right)\left(z / r_{n}\right)^{s_{n}-1}}{\left(1+\left(z / r_{n}\right)^{s_{n}}\right)^{2}}
$$


and

$$
f^{\prime}(z)=\sum_{n=1}^{\infty} f_{n}^{\prime}(z)
$$

We choose $t_{n}$ such that

$$
\left(t_{n} / r_{n}\right)^{s_{n}}=2 .
$$

Suppose that $t_{n} \leqq|z| \leqq \sqrt{r_{n+1}}$. For $k<n$ we get from (10.2) and (10.3)

$$
\left|f_{k}(z)-(-1)^{k}\right|=\left|\frac{1}{1+\left(z / r_{k}\right)^{s_{k}}}\right| \leqq 2\left(r_{k} / r_{n}\right)^{s_{k}}<e^{-k} .
$$

For $k>n$ we get from (10.2) and (10.3)

$$
\left|f_{k}(z)\right| \leqq 2\left(\sqrt{r_{n+1}} / r_{k}\right)^{s_{k}}<e^{-k} .
$$

It follows from (10.5) that $\left|f_{n}(z)\right| \leqq 2$, which implies together with (10.6) and (10.7) that

$$
|f(z)| \leqq 4
$$

for all $z$ lying in $t_{n} \leqq|z| \leqq \sqrt{r_{n+1}}$.

Let $t_{n} \leqq r \leqq \sqrt{r_{n+1}}$. We get from (10.3)

$$
N(r, f) \leqq s_{n} \log \left(r / r_{n}\right)+\log r \sum_{k=1}^{n-1} s_{k} \leqq s_{n} \log \left(r / r_{n}\right)+2 s_{n-1} \log r .
$$

This implies together with (10.8) and (10.4) that

$$
T(r, f) \leqq 2 s_{n} \log r+\log 4 \leqq 2 \varphi\left(\sqrt{r_{n}}\right)(\log r)^{2}+\log 4
$$

for $t_{n} \leqq r \leqq \sqrt{r_{n+1}}$. Since $T(r, f)$ is an increasing function of $r$, we get for $\sqrt{r_{n}}<r<t_{n}$

$$
T(r, f) \leqq T\left(2 r_{n}, f\right) \leqq 2 \varphi(r)\left(\log \left(2 r^{2}\right)\right)^{2}+\log 4,
$$

which together with (10.10) proves (2.10).

From (10.9) and (10.5) we deduce that

and

$$
N\left(t_{n}, f\right) \leqq \log 2+2 s_{n-1} \log t_{n}
$$

$$
N\left(2 t_{n}, f\right) \leqq s_{n} \log 3+3 s_{n-1} \log r_{n} .
$$

These estimates combined with (10.3) and (10.8) yield

$$
T\left(t_{n}, f\right) \leqq 3 s_{n-1} \log r_{n} \leqq s_{n} / n
$$

and

$$
T\left(2 t_{n}, f\right) \leqq 4 s_{n} .
$$


Suppose that $|z|=t_{n}$. We get from (10.5)

$$
\begin{gathered}
\left|f^{\prime}(z)\right| \geqq \frac{s_{n}}{9 r_{n}}-2 \sum_{k<n}\left(s_{k} / r_{k}\right)\left(r_{k} / r_{n}\right)^{s_{k}+1}-2 \sum_{k>n}\left(s_{k} / r_{k}\right)\left(\left(2 r_{n}\right) / r_{k}\right)^{s_{k}-1} \\
\geqq \frac{s_{n}}{9 r_{n}}-\frac{2}{r_{n}} \sum_{k<n} s_{k}-2 \sum_{k>n} s_{k} / r_{k},
\end{gathered}
$$

and we deduce from (10.3) and (10.2) that

$$
\left|f^{\prime}(z)\right| \geqq \frac{s_{n}}{9 r_{n}}-\frac{4 s_{n-1}}{r_{n}}-\frac{4}{\sqrt{r_{n+1}}} \geqq \frac{s_{n}}{18 r_{n}} .
$$

This implies together with (10.8) that

$$
t_{n} \lambda\left(t_{n}, f\right) \geqq \frac{s_{n}}{18 \cdot 17} .
$$

This together with (10.11) and (10.12) proves (2.12) and (2.11). Theorem 6 is proved.

\section{Proof of Theorem 7}

Let $f$ be as in Theorem 7. Let $n(r)$ be defined by (9.1). We have

$$
n(r) \log r \leqq \int_{r}^{r^{2}} n(t) t^{-1} d t \leqq(3+o(1)) T\left(r^{2}, f\right),
$$

and we deduce from (2.13) that there exists $K>0$ such that

$$
n(r)<K \log r
$$

for all large values of $r$. This implies that for any large $r$, there exists $z_{r},\left|z_{r}\right|=r$, such that $f$ does not take any of the values 0,1 and $\infty$ in

$$
\left|z-z_{r}\right|<\frac{r}{K \log r}
$$

and just as in the proof of (9.4), we deduce from (11.1) that

$$
\lambda(r, f)=O\left(\frac{\log r}{r}\right) \text { as } r \rightarrow \infty .
$$

Since $f$ is transcendental, we get

$$
r \lambda(r, f)=O(\log r)=o(T(r, f)) \quad \text { as } \quad r \rightarrow \infty,
$$

which proves Theorem 7 . 


\section{Proof of Theorem 8}

Contrary to the assertion of Theorem 8 , let us suppose that there exists a transcendental meromorphic function $f$ such that

$$
\liminf _{r \rightarrow \infty} \frac{\log \mu(r, f)}{T(r, f)} \leqq-1-9 d
$$

for some $d>0$. Just as in the proof of Theorem 2, we may assume that $f(0)=0$, and deduce (6.3) so that there exist $t>0$ and $s_{0}>0$ such that

$$
m(r, a) \leqq T(r, f)+6+\log (1 / t)
$$

if $0<|a|<9$ and $r>s_{0}$.

It follows from (12.1) that there exists an increasing sequence $r_{n}, r_{n} \rightarrow \infty$ as $n \rightarrow \infty$, such that

$$
\log \varrho(f(z))<-(1+8 d) T\left(r_{n}, f\right)
$$

on $|z|=r_{n}$ for all $n$. Since $m(r, \infty, f) \leqq T(r, f)$ and

$$
m(r, 0, f) \leqq(1+o(1)) T(r, f) \quad \text { as } \quad r \rightarrow \infty,
$$

we may choose a point $z_{n}$ lying on $|z|=r_{n}$ such that

if $n$ is large enough.

$$
|\log | f\left(z_{n}\right)|| \leqq(1+d) T\left(r_{n}, f\right)
$$

Let us suppose that

$$
|\log | f\left(w_{1}\right)||>(1+2 d) T\left(r_{n}, f\right)
$$

for some $w_{1}$ lying on $|z|=r_{n}$. Then we may choose an arc $J_{n}$ contained in $|z|=r_{n}$ such that $z_{n} \in J_{n}$,

$$
|\log | f(w)|| \leqq(1+2 d) T\left(r_{n}, f\right)
$$

for all $w \in J_{n}$, and that

$$
|\log | f\left(w_{k}\right)||=(1+2 d) T\left(r_{n}, f\right)
$$

$(k=2,3)$ for the end points $w_{2}$ and $w_{3}$ of the arc $J_{n}$. For $z \in J_{n}$ we get from (12.3) and (12.6)

$$
\log \left|f^{\prime}(z)\right| f(z) \mid=\log (\varrho f(z))+\log (|f(z)|+1 /|f(z)|) \leqq-(6 d+o(1)) T\left(r_{n}, f\right),
$$

and integrating along $J_{n}$ we deduce that

(12.8) $|\log | f(z) / f\left(z_{n}\right)|| \leqq\left|\int_{z_{n}}^{z} \frac{f^{\prime}(w)}{f(w)} d w\right| \leqq \exp \left\{-(6 d+o(1)) T\left(r_{n}, f\right)\right\}=o(1)$

for all $z \in J_{n}$. This implies together with (12.4) that

$$
|\log | f\left(w_{2}\right)|| \leqq(1+d+o(1)) T\left(r_{n}, f\right) .
$$


This is a contradiction with (12.7) if $n$ is large, and we conclude that

$$
|\log | f(z)|| \leqq(1+2 d+o(1)) T\left(r_{n}, f\right)
$$

for all $z$ lying on $|z|=r_{n}$, and that (12.8) holds for all $z$ lying on $|z|=r_{n}$.

Let us suppose that there exist large values of $n$ such that

$$
\left|f\left(z_{n}\right)\right| \leqq 4 .
$$

Then it follows from (12.8) that $|f(z)| \leqq 8$ on $|z|=r_{n}$, and from (12.3) we get

$$
\log \left|f^{\prime}(z)\right| \leqq-(1+8 d) T\left(r_{n}, f\right)+\log 65
$$

on $|z|=r_{n}$. Integrating along the circle $|z|=r_{n}$, we deduce from (12.11) that

$$
\log \left|f(z)-f\left(z_{n}\right)\right| \leqq-(1+8 d) T\left(r_{n}, f\right)+\log 65+2 \log r_{n}
$$

on $|z|=r_{n}$, which implies that

$$
m\left(r, f\left(z_{n}\right), f\right) \geqq(1+8 d+o(1)) T\left(r_{n}, f\right) .
$$

This contradicts (12.2) and we deduce that (12.10) is not possible if $n$ is large. Therefore we have

$$
\left|f\left(z_{n}\right)\right| \geqq 4
$$

for all large $n$.

It follows from (12.8) that

$$
|f(z)|=(1+o(1))\left|f\left(z_{n}\right)\right|
$$

for all $z$ lying on $|z|=r_{n}$, and we get from (12.12)

$$
\log |f(z)|=(1+o(1)) m\left(r_{n}, \infty, f\right)
$$

for all $z$ lying on $|z|=r_{n}$. This implies together with (12.3) that

$$
\log \left|f^{\prime}(z)\right| \leqq-(1+8 d+o(1)) T\left(r_{n}, f\right)+2 m\left(r_{n}, \infty, f\right)
$$

on $|z|=r_{n}$, and integrating along the circle $|z|=r_{n}$, we get

$$
\log \left|f(z)-f\left(z_{n}\right)\right| \leqq-(1+8 d+o(1)) T\left(r_{n}, f\right)+2 m\left(r_{n}, \infty, f\right)
$$

on $|z|=r_{n}$. Applying the Jensen formula to the function $f(z)-f\left(z_{n}\right)$, we get from (12.14), since $f(0)=0$,

$$
\begin{aligned}
\log \left|f\left(z_{n}\right)\right| & \leqq \frac{1}{2 \pi} \int_{0}^{2 \pi} \log \left|f\left(r_{n} e^{i \alpha}\right)-f\left(z_{n}\right)\right| d \alpha+N\left(r_{n}, \infty, f\right) \\
& \leqq-(1+8 d+o(1)) T\left(r_{n}, f\right)+N\left(r_{n}, \infty, f\right)+2 m\left(r_{n}, \infty, f\right),
\end{aligned}
$$

which together with (12.13) implies that

$$
(1+8 d+o(1)) T\left(r_{n}, f\right) \leqq T\left(r_{n}, f\right) \text { as } n \rightarrow \infty .
$$

This is a contradiction, and we deduce that (3.1) holds for all transcendental meromorphic functions. Theorem 8 is proved. 


\section{Proof of Theorem 9}

Let $f$ be as in Theorem 9. Since

$$
m\left(r, f^{\prime}\right) \leqq m(r, f)+o(T(r, f))
$$

outside a set of finite linear measure, it follows from Lemma 4 that we may choose a sequence $r_{n}, r_{n} \rightarrow \infty$ as $n \rightarrow \infty$, such that

$$
\log |f(z)|=m\left(r_{n}, \infty, f\right)-m\left(r_{n}, 0, f\right)+o\left(T\left(r_{n}, f\right)\right)
$$

and

$$
\begin{aligned}
\log \left|f^{\prime}(z)\right| & =m\left(r_{n}, \infty, f^{\prime}\right)-m\left(r_{n}, 0, f^{\prime}\right)+o\left(T\left(r_{n}, f\right)\right) \\
& \leqq m\left(r_{n}, \infty, f\right)+o\left(T\left(r_{n}, f\right)\right)
\end{aligned}
$$

on $|z|=r_{n}$.

If $m\left(r_{n}, 0, f\right)>0$, we deduce from (13.1) that

$$
m\left(r_{n}, \infty, f\right)=o\left(T\left(r_{n}, f\right)\right),
$$

and if $m\left(r_{n}, \infty, f\right)>0$, then

$$
m\left(r_{n}, 0, f\right)=o\left(T\left(r_{n}, f\right)\right) .
$$

These estimates imply together with (13.1) that

$$
\log \left(1+|f(z)|^{2}\right)=2 m\left(r_{n}, f\right)+o\left(T\left(r_{n}, f\right)\right)
$$

on $|z|=r_{n}$ for all $n$. From (13.2) and (13.3) we get $\log \mu\left(r_{n}, f\right) \leqq-m\left(r_{n}, \infty, f\right)+o\left(T\left(r_{n}, f\right)\right) \leqq-(\delta(\infty, f)+o(1)) T\left(r_{n}, f\right) \quad$ as $\quad n \rightarrow \infty$, which proves Theorem 9.

\section{Proof of Theorem 10}

Let $d$ satisfy $0<d<1$. We set

where $r_{n}=n^{1 / d}$. We have

$$
f(z)=\prod_{n=1}^{\infty} \frac{r_{n}+z}{r_{n}-z}
$$

$$
\frac{f^{\prime}(z)}{f(z)}=\sum_{n=1}^{\infty} \frac{2 r_{n}}{r_{n}^{2}-z^{2}},
$$

and since $|f(i r)|=1$ for any real $r$, we get

$$
\mu(r, f) \geqq \frac{\left|f^{\prime}(i r)\right|}{1+|f(i r)|^{2}} \geqq \sum_{n=1}^{\infty} \frac{r_{n}}{r_{n}^{2}+r^{2}}
$$

for any $r>0$. 
From the choice of $r_{n}$ we deduce that

which implies that

$$
n(r, 0, f)=(1+o(1)) r^{d} \text { as } r \rightarrow \infty,
$$

$$
n(2 r, 0, f)-n(r, 0, f)=\left(2^{d}-1+o(1)\right) r^{d}
$$

as $r \rightarrow \infty$. It follows from (14.1) and (14.2) that

$$
\begin{aligned}
\mu(r, f) \geqq & \sum_{r \leqq r_{n} \leqq 2 r} \frac{r_{n}}{r_{n}^{2}+r^{2}} \geqq(n(2 r, 0, f)-n(r, 0, f)) \frac{r}{5 r^{2}} \\
& \geqq\left(2^{d}-1+o(1)\right) \frac{r^{d}}{5 r} \quad \text { as } \quad r \rightarrow \infty .
\end{aligned}
$$

If $|z|=r$ and $|\arg z|<\pi / 6$, then

$$
\begin{gathered}
\log |f(z)| \geqq \sum_{r \leqq r_{n} \leqq 2 r} \log \left|\frac{r_{n}+z}{r_{n}-z}\right| \\
\geqq(n(2 r, 0, f)-n(r, 0, f)) \log (5 / 4) \geqq\left(2^{d}-1+o(1)\right) r^{d} \log (5 / 4) .
\end{gathered}
$$

This implies that

$$
m(r, \infty, f) \geqq \frac{1}{6} \log (5 / 4)\left(2^{d}-1+o(1)\right) r^{d} .
$$

On the other hand, since

$$
n(r, 0, f)=n(r, \infty, f)=O\left(r^{d}\right) \text { as } \quad r \rightarrow \infty,
$$

we have (see e.g. Nevanlinna [7, p. 223])

$$
T(r, f)=O\left(r^{d}\right) \quad \text { as } \quad r \rightarrow \infty .
$$

From (14.4) and (14.5) we deduce that $\delta(\infty, f)>0$ and that the order of $f$ is $d$. From (14.3) and (14.5) it follows that $f$ satisfies (3.4). Theorem 10 is proved.

\section{Proof of Theorem 11}

Let $f$ be a transcendental meromorphic function. Let $n(r)$ be defined by (9.1). Just as in the proof of Theorem 5 , we deduce that there exists $r_{0}$ such that

$$
n(3 r / 2) \leqq \frac{4 T(2 r, f)}{\log (4 / 3)}
$$

for $r \geqq r_{0}$.

If the lower order of $f$ is infinite, we choose $r_{n}=e^{n}$ for any positive integer $n$. Let us suppose that

$$
\liminf _{r \rightarrow \infty} \frac{T(2 r, f)}{T(r, f)}=\infty
$$


Then we have for any $K>1$,

$$
T(2 r, f)>K T(r, f)
$$

for $r \geqq r_{K}$. This implies that

$$
T\left(2^{n} r_{K}, f\right)>K^{n} T\left(r_{K}, f\right)
$$

for all $n$, and we deduce that if $2^{n-1} r_{K} \leqq t \leqq 2^{n} r_{K}$, then

This implies that

$$
\frac{\log T(t, f)}{\log t} \geqq \frac{(n-1) \log K+\log }{n \log 2+\log \frac{T\left(r_{K}, f\right)}{r_{K}} .}
$$

$$
\liminf _{r \rightarrow \infty} \frac{\log T(r, f)}{\log r}>\log K,
$$

and we deduce that if (15.2) holds, then the lower order of $f$ is infinite. Therefore, if the lower order of $f$ is finite and positive, we may choose a sequence $r_{n}, r_{n} \rightarrow \infty$ as $n \rightarrow \infty$, such that

for all $n$, A being a constant.

$$
\frac{T\left(2 r_{n}, f\right)}{T\left(r_{n}, f\right)}<A
$$

If $f$ satisfies (15.3) for some $K>1$, we deduce from (15.4) that the lower order of $f$ is positive. Therefore, if the lower order of $f$ is zero, then $f$ satisfies (2.3), and it follows from Lemma 3 that there exists a sequence $r_{n}, r_{n} \rightarrow \infty$ as $n \rightarrow \infty$, such that

$$
n\left(3 r_{n} / 2\right)=o\left(T\left(r_{n}, f\right)\right) \text { as } n \rightarrow \infty .
$$

In all cases, we write, if $n\left(r_{n}\right) \geqq 1$,

$$
d_{n}=\frac{r_{n}}{4 n\left(3 r_{n} / 2\right)} .
$$

Then there exists $t_{n}, r_{n} \leqq t_{n} \leqq 3 r_{n} / 2$, such that $f$ does not take any of the values 0,1 and $\infty$ in the annulus

$$
B_{n}=\left\{z: t_{n}-d_{n}<|z|<t_{n}+d_{n}\right\} .
$$

Since the disc $|z-w|<d_{n}$ is contained in $B_{n}$ for all $w$ satisfying $|w|=t_{n}$, we get, just as in the proof of (9.4),

$$
\varrho(f(w)) \leqq \frac{2 K_{1}}{d_{n}}=\frac{8 K_{1} n\left(3 r_{n} / 2\right)}{r_{n}}
$$

for all $w$ lying on $|z|=t_{n}$. This implies that

$$
t_{n} \mu\left(t_{n}, f\right) \leqq 16 K_{1} n\left(3 r_{n} / 2\right) .
$$

Combining (15.1) and (15.7), we get

$$
t_{n} \mu\left(t_{n}, f\right)=O\left(T\left(2 r_{n}, f\right)\right) \text { as } n \rightarrow \infty .
$$


This proves (3.5), since $r_{n} \leqq t_{n} \leqq 2 r_{n}$ and $T(r, f)$ is an increasing function of $r$. Similarly, combining (15.8) and (15.5), we get (3.6) for functions of finite positive lower order. If the lower order of $f$ is zero, then (3.7) follows from (15.7) and (15.6). Theorem 11 is proved.

\title{
References
}

[1] Anderson, J. M., and J. Clunie: Slowly growing meromorphic functions. - Comment. Math. Helv. 40, 1966, 267-280.

[2] Clunie, J., and W. K. Hayman: The spherical derivative of integral and meromorphic functions. - Comment. Math. Helv. 40, 1966, 117-148.

[3] Constantinescu, C.: Einige Anwendungen des hyperbolischen Masses. - Math. Nachr. $15,1956,155-172$.

[4] Hayman, W. K.: Slowly growing integral and subharmonic functions. - Comment. Math. Helv. 34, 1960, 75-84.

[5] LeHto, O.: The spherical derivative of meromorphic functions in the neighbourhood of an isolated singularity. - Comment. Math. Helv. 33, 1959, 196-205.

[6] Lehto, O., and K. I. VirTanen: On the behaviour of meromorphic functions in the neighbourhood of an isolated singularity. - Ann. Acad. Sci. Fenn. Ser. A I Math. 240, 1957, 1-9.

[7] Nevanlinna, R.: Analytic functions. - Die Grundlehren der mathematischen Wissenschaften 162, Springer-Verlag, Berlin-Heidelberg-New York, 1970.

[8] Toppila, S.: Some remarks on exceptional values at Julia lines. - Ann. Acad. Sci. Fenn. Ser. A I Math. 456, 1970, 1-20.

[9] Toppila, S.: Picard sets for meromorphic functions with a deficient value. - Ann. Acad. Sci. Fenn. Ser. A I Math. 5, 1980, 263-300.

[10] Winkler, J.: Zur Existenz ganzer Funktionen bei vorgegebener Menge der Nullstellen und Einsstellen. - Math. Z. 168, 1979, 77-85.

\author{
University of Helsinki \\ Department of Mathematics \\ SF-00100 Helsinki 10 \\ Finland
}

Received 20 May 1981 